\title{
RETRACTED ARTICLE: Neuromodulation of internal emergent representations for sequential tasks
}

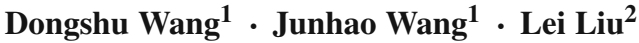

Published online: 16 October 2017

(C) Springer Science+Business Media B.V. 2018

The authors retracted this article because its theoretical basis, some of its text, and some of its figures and tables substantially overlap with the following previously submitted article:

Motivated optimal developmental learning for sequential tasks without using rigid timediscounts, Dongshu Wang, Yihai Duan, Juyang Weng, IEEE Trans. on Neural Networks and Learning Systems, published online 11/01/18, https://doi.org/10.1109/TNNLS.2017. 2762720, http://ieeexplore.ieee.org/document/8253855/.

All authors of this article agree to this retraction. The online version of this article contains the full text of the retracted article as electronic supplementary material.

Electronic supplementary material The online version of this article (https://doi.org/10.1007/s10462017-9585-z) contains supplementary material, which is available to authorized users.

\section{Dongshu Wang}

wangdongshu@zzu.edu.cn

Junhao Wang

630300938@qq.com

Lei Liu

luckyliulei@126.com

1 School of Electrical Engineering, Zhengzhou University, Zhengzhou 450001, Henan, China

2 Department of Research, The People's Bank of China Zhengzhou Sub-Branch, Zhengzhou 450040, Henan, China 